\title{
ECONOMIC SYSTEM DYNAMICS
}

\author{
JOSEPH L. McCAULEY AND CORNELIA M. KÜFFNER
}

Received 4 December 2003

We provide here a qualitative summary of the main ideas from econophysics and finance theory, starting with a thorough criticism of the standard ideas taught in typical economics textbooks. The emphasis is on the Galilean or physicists' approach to market dynamics, as opposed to the standard nonempirical postulatory one.

\section{Static versus dynamic models of pricing}

Standard textbook economic theory quite generally assumes the neoclassical economic model, which is a static model of unregulated free markets. Every western government in our era believes implicitly in a free market with "springs," where imbalances are only of short-term nature. The model postulates the nonempirical idea of utility and assumes stable equilibrium, a notion that goes back over two hundred years to Adam Smith's famous "Invisible Hand."

The standard, reigning theory of free markets asserts that individually selfish behavior can lead collectively to the greatest benefit of society. Modern dynamic approaches, in stark contrast, understand markets as far from equilibrium systems $[6,12]$. How did such fundamentally opposing viewpoints in teaching arise? We first define the basic elements of market dynamics independently of any particular model and then, after surveying the neoclassical model $[27,28]$ critically, we discuss in the other three sections of this paper the empirical basis and implications of market disequilibrium.

To model a market, consider $N$ agents who buy and sell $n$ assets. By agents we mean consumers, producers, traders, and so forth. At any given time $t$ each agent holds quantities $x(t)=\left(x_{1}, \ldots, x_{n}\right)$ of the $n$ different assets. The price of the assets is denoted by $p(t)=\left(p_{1}, \ldots, p_{n}\right)$. The basis for modelling the dynamics of a market of $n$ assets and $N$ traders is the differential equation (or a discrete iterated equation) $d p / d t=\varepsilon(p, t)$ where $\varepsilon(p, t)=D(p, t)-S(p, t)$ is the excess demand calculated for all $N$ agents. Here, $D$ is the total demand and $S$ is the total supply for a given asset "at price $p$ " (in reality there is always a bid/ask spread, so by " $p$ " we mean the price of the last trade). Equilibrium is defined by vanishing excess demand, $\varepsilon=0$. Static models of markets assume a 
dominant stable equilibrium point. This would require a restoring force pulling $p$ back to equilibrium, something like a spring in the market. In other words, the neoclassical model provides not a completely static market but a trivial dynamic system as opposed to chaotic or complex one.

In the neoclassical approach, one begins by postulating a utility function $U(x)$ for each agent. Utility maximization subject to an agent's budget constraint predicts a downward sloping demand curve $p=f(x)$ for the standard case of decreasing returns. Profit maximization or an initial endowment is assumed in order to obtain an upward sloping neoclassical supply curve $p=g(x)$. These functions must then be inverted to obtain the demand and supply schedules $x=D(p)$ and $x=S(p)$ required by dynamics. Here, the dynamic approach to equilibrium does not represent trading, but only represents a "price-adjustment auction," as was proposed by Leon Walras.

The statics of the neoclassical model lies in the assumption that there is no trading at all unless and until all prices reach equilibrium, at which point all sellers and buyers simply exchange goods. In other words, the neoclassical theory provides only a model of a pure barter economy where the economic efficiency $e=\min (S / D, D / S)$ is fixed artificially at $100 \%$. The meaning of the model is that every agent who wants to trade does so. There is no excess demand or supply, no shortage of labor, goods or services, and no unemployment. Arrow and Hurwicz [2] have shown that the condition for the mathematical existence of a neoclassical equilibrium is that all agents have the same perfect information all the way into the infinite future, and conform to the same view of the future. Money does not and cannot appear: there is no capital accumulation, there is no demand for financial markets: the price $p$ in the model is only a label [8]. This means that the variable $p$ does not represent any physical or informational quantity. It does not represent a piece of paper or a piece of metal, bits in computer memory, or even an unspoken obligation to pay something at a later time. In other words, it represents no aspect that is characteristic of "money."

The first econophysicist, Osborne [18], tried hard to make sense of textbook economic theory, and consequently pointed out that the neoclassical supply-demand curves do not occur empirically in microeconomic reality. He explained that price as a function of demand $p=f(x)$ does not exist mathematically because the observable quantity, demand as a function of price $x=D(p)$, is noninvertible because it is a step function. There is a connection with nontrivial dynamics. Unknown to Osborne, Hamiltonian models were used in economics in applications to markets of the theory of optimization and control. In a nonintegrable Hamiltonian system, the analog of Osborne's microeconomic result is that the canonical momentum $p=f(q)$ does not exist as a function of generalized coordinate $q$. This was first noted by Einstein in 1917 in the context of explaining why the threebody problem and mixing systems are not susceptible to Bohr-Sommerfeld quantization rules [11]. This explains Osborne's empirical observation from a theoretical standpoint, but only for economic models defined by optimization and control via maximizing a utility functional. In the nonintegrable case, a utility function and stable equilibria do not exist. There are only a path-dependent functional $U$ and unstable equilibria (hyperbolic points). The utility functional coincides with the action in Hamiltonian dynamics [12]. 
Another interesting inconsistency in the standard textbook model was uncovered by Sonnenschein $[23,24]$, who showed that if one starts with individual agents who maximize utility locally (neoclassical microeconomic model), then aggregation over all agents does not lead to a neoclassical macroeconomic model (downward-sloping demand curve $p=f(x)$ ) or to any definite supply-demand curves at all: any curves at all or none are possible. In other words, combining the results of Sonnenshein and Osborne, we can say that there is no theoretical or empirical underpinning for the neoclassical macroeconomic intersecting supply-demand curves portrayed nonempirically in every standard economics text.

From the standpoint of econophysics, which requires empirically grounded and falsifiable models of markets, we see that the neoclassical economic model has been falsified both on the micro- and macroeconomic scales: none of its predictions has been found to be even approximately true. Yet, the World Bank, the IMF, the US Treasury, and the EU persist in applying the model to formulate requirements to be met by Third World countries to obtain loans [26], and to so-called First World countries, for example, to demand that agricultural subsidies be eliminated with the expectation of improving market efficiency. The neoclassical model teaches equilibrium and stability whereas Soros [25] and Stiglitz [26] have observed qualitatively, based on experience, that markets are unstable.

But game theory [19] is also used in economic theorizing and predicts equilibria, so it is necessary to consider that approach as well. The neoclassical notion of expected utility was invented by D. Bernoulli in an attempt to resolve the St. Petersburg paradox. Expected utility was later used by John von Neumann and Oscar Morgenstern to formulate the theory of cooperative games. Much later still came a class of neoclassical equilibrium models with efficiency less than $100 \%$, called "asymmetric information," that assumes that sellers know more than buyers. From this standpoint, an equilibrium based on expected utility maximization is predicted in the "lemon trap model" invented by Akerlof [1], which was used later by Krugman [10] to discuss the idea of Keynesian liquidity traps. But there is no dynamics in the asymmetric information model, and there is also no empirical evidence for the equilibrium predicted by the model. The model does take a qualitative step in the right direction by avoiding the completely unrealistic assumption of perfect information and infinite foresight by all agents, all of whom are economic clones of a single "infinitely rational" agent.

Von Neumann and Morgenstern's original game theoretic results are effectively confined to two players. Three or more players were treated only in a mean field approximation, which is unrealistic. John Nash later discovered a class of neoclassical equilibria not included by von Neumann and Morgenstern in game theory, whereby agents exhibit selfish behavior and are supposed to have no regrets after playing the game. Nash's aim was to model Adam Smith's Invisible Hand. However, there is no empirical evidence for Nash equilibria. Nash equilibria require "defection" rather than "cooperation" in the prisoner's dilemma for example, and in other noncooperative games. In order to make an empirical test, good statistics are required, but when the prisoner's dilemma is played repeatedly, yielding the iterated prisoner's dilemma, then the agents tend to cooperate rather than defect. This was first shown by the famous Dresser experiment. Von Neumann later 
abandoned game theory as a tool for social and economic modelling in favor of the study of automata [14].

Fortunately, there is a way to test any set of market price data empirically for the influence of Adam Smith's Invisible Hand. The implication of the Invisible Hand is that a stable equilibrium occurs. Empirically, noise should cause small fluctuations about this equilibrium, or at least about a steady state, and in this way one can either confirm or falsify the assumption of equilibrium. Therefore, one needs only to test observed time series of prices $p(t)$ for stationarity [12]. To date, there is no convincing evidence for stationarity of any known price data from real markets. In particular, observed financial market distributions, both empirically and in realistic models, are very far from stationarity.

\section{Deterministic models of economic systems}

The first purely theoretical illustration of the lack of internal consistency dynamically within the neoclassical model was produced by Scarf [22]. He constructed a model utility whereby three agents exchange three assets. Scarf then showed that instead of equilibrium one finds a stable limit cycle where, according to the neoclassical assumptions, trading is never allowed. It shows that production also is not described, all that is traded is the "initial endowment" of each agent. Later, Saari [21] pointed out that excess demand functions that disobey Arrow's restrictions will typically produce nonintegrable, chaotic models.

More interesting is that Radner [20] showed that, even though the neoclassical equilibrium is formally mathematically guaranteed "to exist," if one introduces the slightest uncertainty, then the agents cannot compute/locate that equilibrium. So, complexity shows its face as soon as one tries to introduce uncertainty into the neoclassical equilibrium model; Radner's proof of lack of computability of equilibrium via a Turing machine is equivalent to maximum computational complexity. Money and financial markets do not appear in the equilibrium model, where goods are merely exchanged. Where and how, then, might "money" enter into the picture? Kirman [8] has suggested that demand for money, in other words liquidity demand, does not appear in the usual neoclassical equilibrium model because the future is completely determined. Kirman speculates that liquidity demand and the consequent supply of money should arise from uncertainty: without uncertainty about how to list and cover one's future needs, money is unnecessary.

Can one derive the demand for money by relaxing any of the assumptions slightly within a neoclassical setting? The bounded rationality model of Bak et al. [3] attempts to do exactly that. In agreement with Kirman, something suggestive of money appears transiently in Bak's utility maximization model after adding uncertainty in the form of noise, but markets still are cleared at the end of each trading day as in the original noisefree model. Therefore, capital and capital accumulation in the form of financial markets still do not appear. There is, to date, no utility-based model that includes the possibility of financial markets, and financial markets now dominate the world. In addition, business cycles, market bubbles, crashes, and all other dynamic market activities are impossible to discuss meaningfully from within the neoclassical picture, which we now abandon in favor of empiric reality. 
The basic aim in economics still is to replicate the original financial market success of Osborne [5], and Black and Scholes [4]: to construct a falsifiable economic model that captures the main features of some particular market over a reasonable era. In the face of all the thick economics texts on library shelves, this problem stands completely unsolved.

\section{Business cycles}

Business cycles [5] would require either periodicity or approximating quasiperiodicity. The best bet for a model of a business cycle, if a business cycle exists, might be a stable but noisy limit cycle. Evidence for periodicity in market data was searched for by Maurice Kendall and by Clive Granger and Oscar Morgenstern but no evidence for stable or neutral periodicity was found. We know now that stable periodicity requires negative Liapunov exponents; neutrally stable motion corresponds to vanishing exponents.

Deterministic chaos is pseudorandom via unstable periodicity and unstable quasiperiodicity. In the 1980's, the heyday of nonlinear dynamics, evidence for deterministic chaos was sought in economic data in the search for empirical evidence for positive Liapunov exponents. Again, the results were negative. To date, no approximately correct and falsifiable deterministic model of market dynamics exists.

Falsifiable stochastic models of financial markets are available, but show no evidence for a business cycle, or for any market periodicity at all. The problem with trying to model business cycles is the lack of hard empirical evidence for the notion of any evident periodicity/regularity.

Markets go up and markets go down, but with no apparent regularity. Therefore, under the influence of statistical physicists and nonlinear dynamicists working in economics and finance ("econophysicists"), the emphasis in the last decade has switched from looking for business cycles to the attempt to explain the dynamics of the large price movements that are clearly indicated by financial market statistics. These large fluctuations are characterized by the so-called "fat tails" in the returns distribution [12]. Let $p(t)$ denote the price of an asset at time $t$. The idea of a fat-tailed probability density $f(x)$ of returns $x=\ln p(t) / p(0)$ is that for large magnitude returns one observes $f(x) \approx x^{-\mu}$, where $\mu$ is the "tail exponent." Fat-tailed returns distributions are very easy to model mathematically, therefore the problem is how to eliminate the nonuniqueness, the ability to derive exactly the same tail exponent from many different dynamical models not included in any well-defined universality class, and arrive at a mathematical model that reflects approximately what a given market is really doing dynamically. This leads us to stochastic models of financial markets.

\section{Stock market dynamics}

The first stochastic model of asset pricing obeying the constraint of nonnegative prices is Osborne's lognormal model of prices [5]. There, the return $x=\ln p(t) / p(0)$ is assumed to be Gaussian. Since prices are unbounded in the absence of price controls, Osborne's lognormal model of the price distribution is nonstationary: the Gibbs entropy of the distribution increases without bound. The lognormal pricing model was used by Fischer 
Black and Myron Scholes some fifteen years after Osborne's discovery in order to construct the first falsifiable model of option pricing. The model fails (honorably) to describe asset prices correctly because large returns $x$ occur with much greater frequency than can be explained by the Gaussian distribution.

There was once hope, inspired by Benoit Mandelbrot [5], that Levy distributions could be applied to financial markets, but the range of observed (nonuniversal) tail exponents $\mu$ of empirical financial distributions is much too large for the Levy distribution to be of interest. For small-to-moderate returns, the empirical distribution is exponential in $x$ $[12,13]$, the distribution is not approximately Gaussian or Levy. The exponential distribution has interesting stochastic dynamics. A diffusion coefficient that is linear in $(x-R t) / \sqrt{t}$ is implied in a Markovian model, where $R=\langle\ln p(t) / p(0)\rangle$ is the expected return. Good agreement with option pricing by traders has been obtained by using this model. Again, the model is far from stationarity. Adam Smith's Invisible Hand is apparently completely unreliable in financial markets, which have no equilibria and certainly no springs.

The deduction of market dynamics from empirical data is not unique due to the limitations of finite precision in measurement. The nonuniqueness occurs in the following way. For example, there are infinitely many different Markov models that generate the observed time dependence of the exponential distribution, but (fortunately) it has been shown that those models cannot be distinguished from each other on a trading time scale that is small compared with one hundred years. The greater problem is how to model nonfinancial markets in any meaningful way. The worse the data, the greater is the problem of nonuniqueness in deducing the dynamics empirically.

Nonuniqueness in deducing dynamics from data is a lesson that is anticipated from recent experience in nonlinear dynamics, where if a chaotic system has a generating partition, then the partition (and not statistics) characterizes the dynamical model [7]. To determine which chaotic model generated a particular observed time series, then the generating partition must be extracted to within relatively high numerical precision from that series. This was found to be quite difficult for empirical data at the transition to turbulence, but no simpler alternative is available.

Moore $[15,16,17]$ has explained implicitly why we should have no hope to be able to deduce dynamics of complexity from truly complex empirical data: a complex deterministic system has no generating partition. In the spirit of modern nonlinear dynamics, Moore went on to present examples of one- and two-dimensional iterated maps that are Turing machine-equivalent [9]. Financial data have been modelled so far by noncomplex stochastic dynamics. The complexity is hidden in part in the expected return $R$, which can change suddenly and which we do not know how to model in any useful way.

Summarizing, no falsifiable, approximately correct dynamic model of any nonfinancial market exists. The multitude of "graphs" presented without error bars in current economics texts are not better than cartoons because they are not based on real empirical data, only on falsified neoclassical expectations. This is good news for econophysicists and nonmainstream economists: they can analyze the available economic data and try to rewrite the economics texts, if they want to take on that challenge. The goal is to 
make nonfinancial economic modelling more like present-day finance modelling, which is firmly empirically grounded.

\section{References}

[1] G. A. Akerlof, An Economic Theorist's Book of Tales, Cambridge University Press, Cambridge, 1984.

[2] K. J. Arrow and L. Hurwicz, On the stability of the competitive equilibrium. I, Econometrica 26 (1958), 522-552.

[3] P. Bak, S. F. Nørrelykke, and M. Shubik, The Dynamics of Money, in press.

[4] F. Black and M. Scholes, The pricing of options and corporate liabilities, J. Pol. Econ. 81 (1973), no. 3, 637-654.

[5] P. Cootner, The Random Character of Stock Market Prices, MIT Press, Massachusetts, 1964.

[6] G. Dosi, Innovation, Organization and Economic Dynamics: Selected Essays, Edward Elgar Publishing, Cheltenham, 2001.

[7] G. H. Gunaratne, Universality beyond the onset of chaos, Chaos (Woods Hole, Mass, 1989), Amer. Inst. Phys., New York, 1990, pp. 37-69.

[8] A. Kirman, The intrinsic limits of modern economic theory: the emperor has no clothes, Econom. J. 99 (1989), no. 395, 126-139.

[9] P. Koiran and C. Moore, Closed-form analytic maps in one and two dimensions can simulate universal Turing machines, Theoret. Comput. Sci. 210 (1999), no. 1, 217-223.

[10] P. R. Krugman, The Return of Depression Economics, W. W. Norton, New York, 2000.

[11] J. L. McCauley, Classical Mechanics. Transformations, Flows, Integrable and Chaotic Dynamics, Cambridge University Press, Cambridge, 1997.

[12] Dynamics of Markets: Econophysics and Finance, Cambridge University Press, Cambridge, 2004.

[13] J. L. McCauley and G. H. Gunaratne, An empirical model of volatility of returns and option pricing, Phys. A 329 (2003), no. 1-2, 178-198.

[14] P. Mirowski, Machine Dreams: Economics Becomes a Cyborg Science, Cambridge University Press, Cambridge, 2002.

[15] C. Moore, Unpredictability and undecidability in dynamical systems, Phys. Rev. Lett. 64 (1990), no. 20, 2354-2357.

[16] _ Generalized one-sided shifts and maps of the interval, Nonlinearity 4 (1991), no. 3, $727-745$.

[17] _ Generalized shifts: unpredictability and undecidability in dynamical systems, Nonlinearity 4 (1991), no. 2, 199-230.

[18] M. F. M. Osborne, The Stock Market and Finance from a Physicist's Viewpoint, Crossgar Press, Minneapolis, 1977.

[19] W. Poundstone, Prisoner's Dilemma, Anchor Books, New York, 1992.

[20] R. Radner, Competitive equilibrium under uncertainty, Econometrica 36 (1968), 31-58.

[21] D. Saari, Mathematical complexity of simple economics, Internat. Econom. Rev. 1 (1960), 157.

[22] H. Scarf, Some examples of global instability of the competitive equilibrium, Internat. Econom. Rev. 1 (1960), 157-172.

[23] H. Sonnenschein, Market excess demand functions, Econometrica 40 (1972), 549-563.

[24] Do Walras' identity and continuity characterize the class of community excess demand functions? J. Econom. Theory 6 (1973), no. 4, 345-354.

[25] G. Soros, The Alchemy of Finance: Reading the Mind of the Market, John Wiley \& Sons, New York, 1994.

[26] J. E. Stiglitz, Globalization and Its Discontents, W. W. Norton, New York, 2002.

[27] H. R. Varian, Microeconomic Analysis, W. W. Norton, New York, 1992. 


\section{Economic system dynamics}

[28] Intermediate Microeconomics: A Modern Approach, W. W. Norton, New York, 1999.

Joseph L. McCauley: Department of Physics, University of Houston, Houston, TX 77204, USA; Tiefetfeld Street 14, A6632 Ehrwald, Ausrtia

E-mail address: jmccauley@uh.edu

Cornelia M. Küffner: Tiefetfeld Street 14, A6632 Ehrwald, Austria 


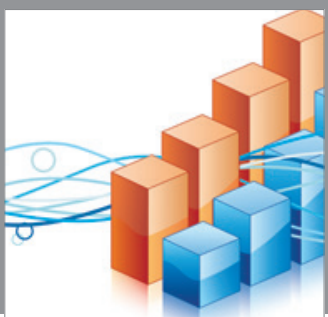

Advances in

Operations Research

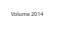

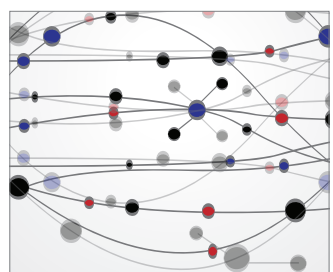

\section{The Scientific} World Journal
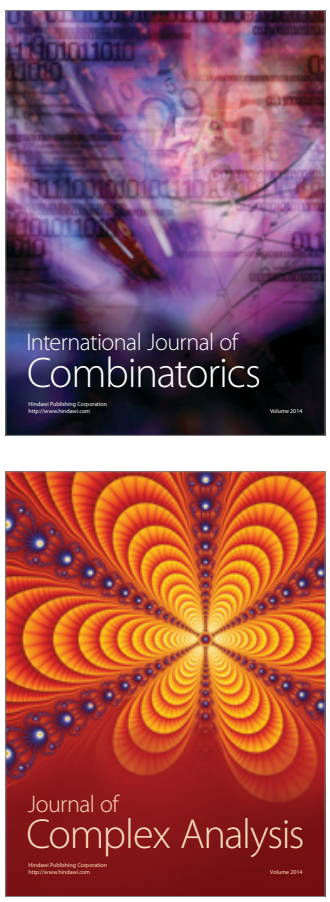

International Journal of

Mathematics and

Mathematical

Sciences
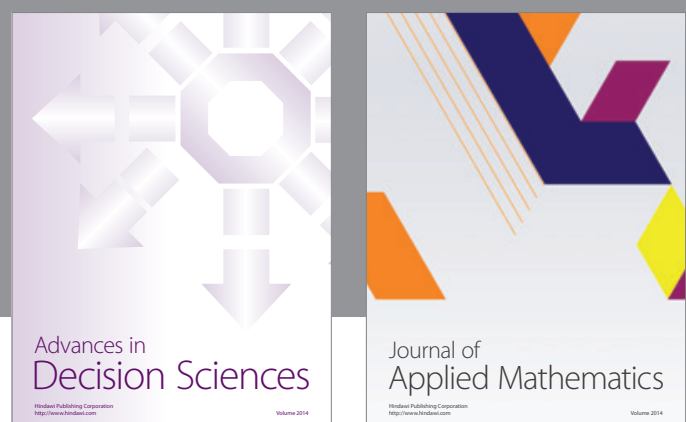

Journal of

Applied Mathematics
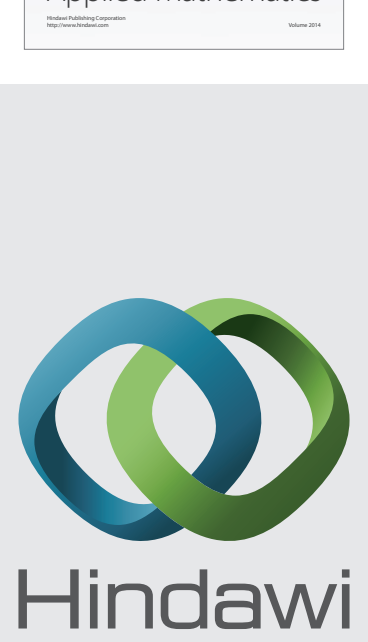

Submit your manuscripts at http://www.hindawi.com
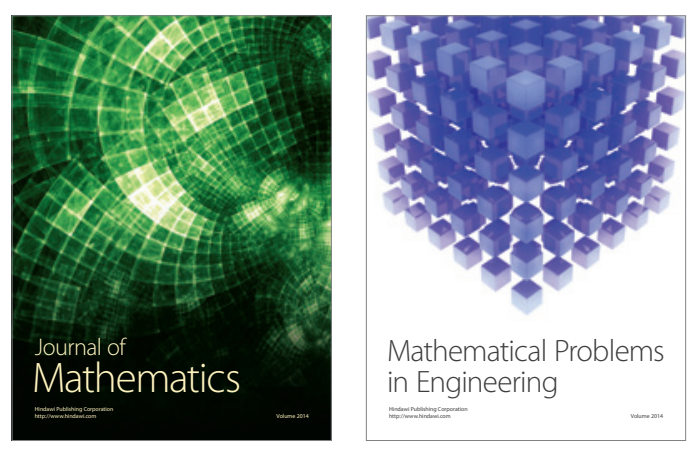

Mathematical Problems in Engineering
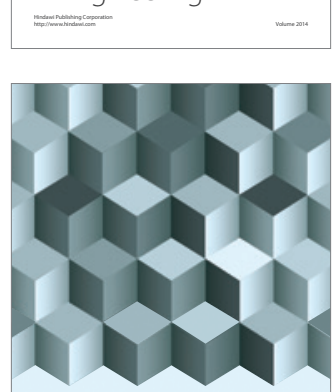

Journal of

Function Spaces
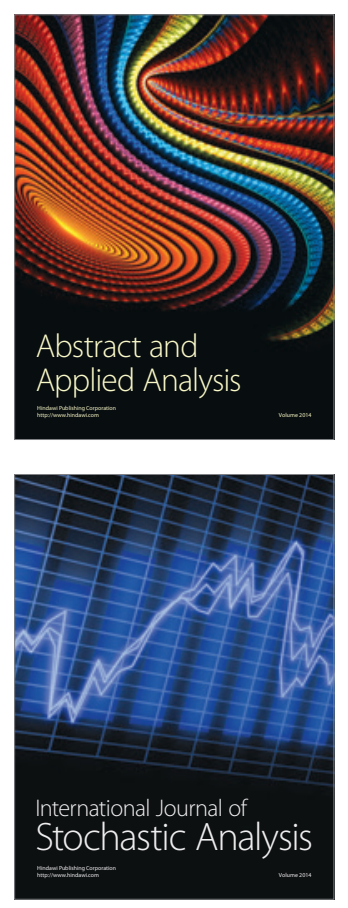

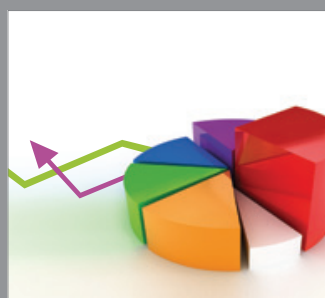

ournal of

Probability and Statistics

Promensencen
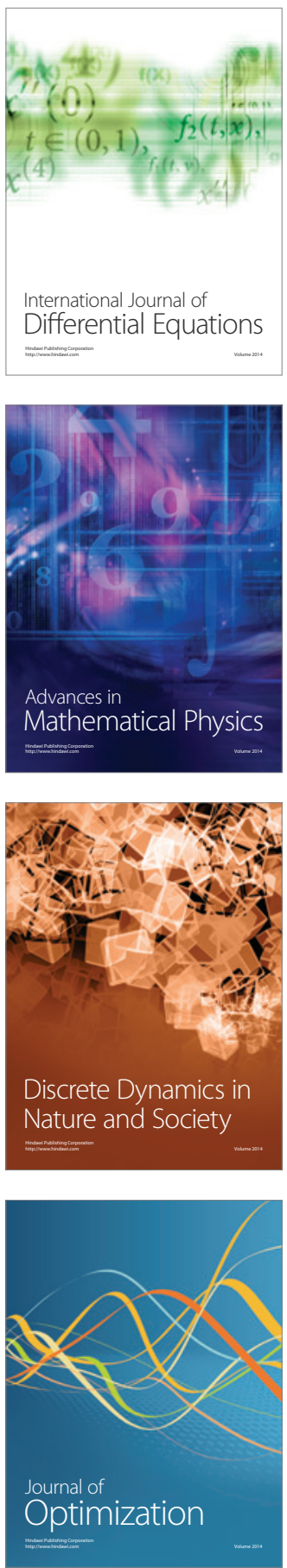